\title{
Comparison of Piperacillin and Penicillin in the Treatment of Uncomplicated Gonorrhea
}

\author{
MARGARET L. SIMPSON, ${ }^{1}$ MOHAMMED Y. KHAN, ${ }^{1 *}$ YOUSUF SIDDIQUI, ${ }^{1}$ ROBERT P. \\ GRUNINGER, ${ }^{2}$ AND DAVID I. WIGREN ${ }^{3}$ \\ Department of Medicine, Section of Infectious Diseases, ${ }^{1}$ Departments of Pathology ${ }^{2}$ and Obstetrics- \\ Gynecology, ${ }^{3}$ Hennepin County Medical Center, University of Minnesota Medical School, Minneapolis, \\ Minnesota 55415
}

Received 4 December 1981/Accepted 4 February 1982

\begin{abstract}
A total of 120 men with uncomplicated infections caused by $\beta$-lactamasenegative, highly penicillin-susceptible strains of Neisseria gonorrhoeae were included in this study. They were randomly assigned to regimens of either piperacillin $(2.0 \mathrm{~g})$ or procaine penicillin $\mathrm{G}\left(4.8 \times 10^{6} \mathrm{U}\right)$ intramuscularly, both delivered concomitantly with an oral dose of $1.0 \mathrm{~g}$ probenecid. A total of 103 patients completed the study, providing data from 112 infected sites: for the penicillin regimen-urethra, 46; pharynx, 5; and rectum, 4; for the piperacillin regimen-urethra, 53; pharynx, 3; and rectum, 1 . In the penicillin group, there were no failures at any of the infected sites. In the piperacillin group, all except one pharyngeal infection were cured. Also, in the piperacillin group, four men returned with positive pharyngeal cultures which had been sterile on the initial visit, whereas no cases of this type occurred in the penicillin group. No major side effects were noted in either group. Clinically, piperacillin was as effective as procaine penicillin $\mathrm{G}$ in the treatment of gonococcal urethritis in men. Pharyngeal infection may be refractory to piperacillin therapy.
\end{abstract}

Piperacillin, a new piperazine penicillin, has been shown to be the most active penicillin against Neisseria gonorrhoeae, including $\beta$-lactamase-positive strains (9). Of the $\beta$-lactamasenegative strains, $90 \%$ was inhibited by piperacillin at a concentration of $0.03 \mu \mathrm{g} / \mathrm{ml}$ (range, $\leq 0.008$ to 0.06 ) compared with $0.5 \mu \mathrm{g} / \mathrm{ml}$ (range, 0.008 to 1.0$)$ for penicillin. With $\beta$-lactamasepositive strains, the concentration effective against $90 \%$ of the isolates was $1.5 \mu \mathrm{g} / \mathrm{ml}$ (range 0.12 to 2.0 ) for piperacillin and $6.0 \mu \mathrm{g} / \mathrm{ml}$ (range 0.5 to 8.0 ) for penicillin (9). The mean peak serum concentration of piperacillin after a $2.0-\mathrm{g}$ intramuscular injection has been reported to be $30.2 \mu \mathrm{g} / \mathrm{ml}$, with a half-life of $80.6 \mathrm{~min}(10)$. By giving $1.0 \mathrm{~g}$ of probenecid orally before the intramuscular injection, the peak serum concentration could be increased and the half-life prolonged by $30 \%$ (10). This study was undertaken to compare the efficacy and safety of piperacillin with that of penicillin in the single-dose treatment of uncomplicated gonococcal infections.

(This work was presented at the 21st Interscience Conference on Antimicrobial Agents and Chemotherapy, M. L. Simpson, M. Y. Khan, Y. Siddiqui, R. P. Gruninger, and D. I. Wigren, Program Abstr. Intersci. Conf. Antimicrob. Agents Chemother. 21st, Chicago, Ill., abstr. no. 613,1981 .)

\section{MATERIALS AND METHODS}

Patient selection. Only male patients were enrolled in the study. All patients were seen between July, 1980, and April, 1981, at the Venereal Disease Clinic affiliated with Hennepin County Medical Center. Patients were eligible for inclusion in the study if urethral smears demonstrated gram-negative intracellular diplococci or if previous pharyngeal or rectal cultures were positive. Informed, written consent was obtained from all participating patients. Reasons for exclusion from the study were presence of a serious underlying disease, evidence of syphilis or disseminated gonococcal infection, known allergies to penicillin, lidocaine, or probenecid, or the use of antibiotics in the previous 10 days.

Treatment and follow-up. Patients were assigned to one of the two treatment regimens by an allocation schedule of random numbers. The first regimen consisted of $2.0 \mathrm{~g}$ of piperacillin reconstituted with $6.6 \mathrm{ml}$ of lidocaine. The alternate regimen was $4.8 \times 10^{6} \mathrm{U}$ of aqueous procaine penicillin. Doses of both drugs were divided and administered intramuscularly at two sites in the gluteal muscle mass. Both groups concomitantly received $1.0 \mathrm{~g}$ of probenecid orally. After examination by a physician, the participants were treated. Reevaluation and test of cure cultures were obtained 3 to 7 days after treatment. Participants were asked to refrain from sexual intercourse between the time of treatment and the follow-up evaluation.

Laboratory methods. All culture specimens were immediately inoculated on Thayer-Martin media and then incubated at $35^{\circ} \mathrm{C}$ in an atmosphere with air and 
$5 \% \mathrm{CO}_{2}$. Colonies of $N$. gonorrhoeae were identified by typical morphological appearance, Gram-stain, positive oxidase test, and carbohydrate utilization tests. The $N$. gonorrhoeae isolates were tested for beta-lactamase by an acidometric method (8). Heavy suspensions of pure cultures were made in Trypticase soy broth (BBL Microbiology Systems, Cockeysville, Md.) containing $20 \%$ glycerol and were immediately stored at $-70^{\circ} \mathrm{C}$ for future use.

Piperacillin was obtained from Lederle Laboratories, Pearl River, N.Y., and penicillin G was obtained from Wyeth Laboratories, Philadelphia, Pa.

The minimum inhibitory concentrations of piperacillin and penicillin for 108 clinical isolates from the study population were determined by the agar dilution technique (4).

Pre- and posttreatment laboratory tests included complete blood counts, platelet count, urinalysis, blood urea nitrogen, creatinine, alkaline phosphatase, serum glutamic oxaloacetic transaminase, bilirubin, and serology for syphilis.

\section{RESULTS}

A total of 120 men were enrolled in the study. Ten patients with sterile pretreatment cultures and seven patients who did not return for the follow-up examination were excluded. Of the remaining 103 patients, 54 were treated with piperacillin, and 49 received penicillin. Age, race, and duration of symptoms were comparable in the two groups. The total number of infected sites was 112 .

Results of the two treatment groups are summarized in Table 1 . In the penicillin group, there were no failures at any of the infected sites. Piperacillin cured all 53 urethritis cases and the single rectal infection. However, one of the three pharyngeal infections was not eradicated by piperacillin. Also, after therapy with piperacillin, four men returned with positive pharyngeal cultures which had been sterile on the initial visit. In these patients, oral sexual exposure between the time of treatment and the follow-up evaluation could not be excluded with certainty. No cases of this type occurred in the penicillin group. No $\beta$-lactamase-positive $N$. gonorrhoeae strains were found in this study.

No major adverse side effects were noted in either treatment group.

Eosinophilia (range, 6 to $8 \%$ ) was found on the follow-up visit in four patients receiving piperacillin and in two patients receiving penicillin. Two patients in each group had elevated levels of serum glutamic oxalacetic transaminase on the return visit (range, 70 to $96 \mathrm{U}$, with a normal of up to $40 \mathrm{U} /$ /iter). The patients exhibiting abnormal laboratory values were not available for further evaluation. Severe pain at the injection sites was noted by one patient receiving piperacillin. Two men in the piperacillin group and three men in the penicillin group were treated for postgonococcal urethritis.
TABLE 1. Treatment results with 103 male patients (112 infection sites)

\begin{tabular}{lcc}
\hline \multirow{2}{*}{ Infection site } & \multicolumn{2}{c}{ No. cured/no. treated } \\
\cline { 2 - 3 } & $\begin{array}{c}\text { Piperacillin } \\
\text { group }\end{array}$ & $\begin{array}{c}\text { Penicillin } \\
\text { group }\end{array}$ \\
\hline Urethra & $53 / 53$ & $46 / 46$ \\
Pharynx & $2 / 3$ & $5 / 5$ \\
Rectum & $1 / 1$ & $4 / 4$ \\
\hline
\end{tabular}

The minimal inhibitory concentrations for piperacillin and penicillin against the $108 \beta$-lactamase-negative $N$. gonorrhoeae isolated from the study population are given in Fig. 1. Of these isolates, $90 \%$ were inhibited by $\leq 0.03 \mu \mathrm{g}$ of piperacillin per ml compared with $0.125 \mu \mathrm{g}$ of penicillin per $\mathrm{ml}$. The range of minimal inhibitory concentrations for penicillin was $<0.03$ to $0.25 \mu \mathrm{g} / \mathrm{ml}$ compared with $<0.03$ to $0.5 \mu \mathrm{g} / \mathrm{ml}$ for piperacillin.

\section{DISCUSSION}

Since its discovery, penicillin has been the primary antimicrobial used in the single-dose treatment of gonorrhea. During this time, the susceptibility of gonococci to penicillin has decreased (6), and $\beta$-lactamase-positive strains highly resistant to penicillin have emerged $(3,4)$. This has initiated a search for new antimicrobial agents that can be used safely in the single-dose treatment of gonorrhea.

Several new beta-lactam antimicrobials have shown excellent in vitro activity against $N$. gonorrhoeae, including $\beta$-lactamase-positive strains $(7,9)$. Cefoxitin has been shown to cure $100 \%$ of gonococcal urethritis cases, including infections caused by $\beta$-lactamase-positive strains (1). In the same study, penicillin cured

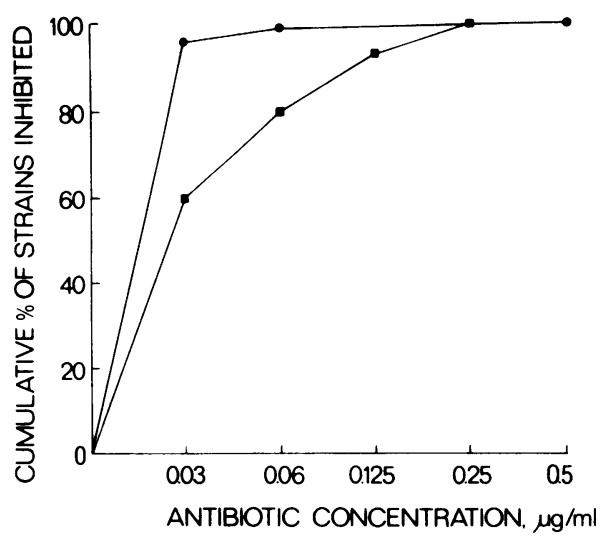

FIG. 1. Comparison of the minimum inhibitory concentrations of piperacillin $(O)$ and penicillin $(\square)$ for $108 N$. gonorrhoeae strains isolated from the study population. 
$96 \%$ of the $\beta$-lactamase-negative infections, but only $23 \%$ of the $\beta$-lactamase-positive infections. In another study, $1.0 \mathrm{~g}$ of cefotaxime cured all cases of urethritis caused by $\beta$-lactamase-positive strains compared with a $19 \%$ cure rate for penicillin (D. J. Lancaster, S. W. Berg, and W. O. Harrison, Program Abstr. Intersci. Conf. Antimicrob. Agents Chemother. 20th, New Orleans, La., abstract no. 675, 1980). Early in vitro data showed piperacillin to have good activity against both $\beta$-lactamase-negative and -positive $N$. gonorrhoeae (9). A clinical study in the Philippines demonstrated the efficacy of piperacillin with $99 \%$ cure rates in the therapy of gonococcal cervicitis caused by both $\beta$-lactamase-positive and -negative strains (L. VirayZerbo, M. Del Casal, and P. G. Gooding, Program Abstr. Intersci. Conf. Antimicrob. Agents Chemother. 20th, New Orleans, La., abstract no. 482,1980$)$. However, in a recently reported study from the United States, piperacillin cured $97 \%$ of the cases of gonococcal urethritis caused by $\beta$-lactamase-negative strains compared with $78 \%$ for $\beta$-lactamase-positive strains. (D. J. Lancaster, F. S. Wignall, S. B. J. Kerbs, W. O. Harrison, and S. W. Berg, Program Abstr. Intersci. Conf. Antimicrob. Agents Chemother. 21st, Chicago, Ill., abstract no. 406, 1981). These data suggest that piperacillin, in contrast to the new cephalosporins, is a less effective antimicrobial in gonococcal infections caused by $\beta$-lactamase-positive strains.

Our results are in agreement with the recent report of Landis et al. (5), which also showed $100 \%$ cure rates for both penicillin and piperacillin in urethritis caused by $\beta$-lactamase-negative $N$. gonorrhoeae. However, treatment results for gonococcal pharyngeal infection are not reported in their study. Piperacillin failed to eradicate pharyngeal infection in one of three patients in our study, whereas penicillin cured all five patients. Furthermore, four piperacillin-treated patients with initial negative pharyngeal cultures returned with positive cultures for $N$. gonorrhoeae. In one reported study, $22 \%$ of the patients with gonococcal pharyngeal infections presented with negative initial cultures (2). A second culture is required to identify all cases of pharyngeal gonococcal infections. The above four patients were either reinfected, or their pharyngeal infection was not detected at the time of their first visit, and piperacillin failed to eradicate this site of infection.

In summary, piperacillin appears to be an effective antimicrobial agent in treating gonococcal urethritis caused by $\beta$-lactamase-negative strains. For pharyngeal and rectal infections, more clinical data are needed before conclusions can be drawn.

\section{ACKNOWLEDGMENTS}

This investigation was supported by a grant from Lederle Laboratories.

We acknowledge the assistance of the medical personnel at the Red Door Clinic, Hennepin County Medical Center. Sue Counter assisted in the preparation of the manuscript.

\section{LITERATURE CITED}

1. Berg, S. W., M. E. Kilpatrick, W. O. Harrison, and J. A. McCutchan. 1979. Cefoxitin as a single-dose treatment for urethritis caused by penicillinase-producing Neisseria gonorrhoeae. N. Engl. J. Med. 301:509-511.

2. Bro-Jorgensen, A., and T. Jensen. 1973. Gonococcal pharyngeal infections-report of 110 cases. Br. J. Vener. Dis. 49:491-499.

3. Jaffe, H. W., J. W. Biddle, S. R. Johnson, and P. J. Wiesner. 1981. Infections due to penicillinase producing Neisseria gonorrhoeae in the United States: 1976-1980. J. Infect. Dis. 144:191-197.

4. Jaffe, H. W., J. W. Biddle, C. Thornsberry, R. E. Johnson, R. E. Kaufman, G. H. Reynolds, and P. J. Wiesner and the Cooperative Study Group. 1976. National gonorrhea therapy monitoring study; in vitro antibiotic susceptibility and its correlation with treatment results. N. Engl. J. Med. 294:5-9.

5. Landis, S. J., R. Ramphal, B. J. Mansheim, K. H. Rand, and J. W. Shands, Jr. 1981. Comparative efficacy of piperacillin and penicillin $G$ in treatment of gonococcal urethritis. Antimicrob. Agents Chemother. 20:693-695.

6. Martin, J. E., Jr., A. Lester, E. V. Price, and J. D. Schmale. 1970. Comparative study of gonococcal susceptibility to penicillin in the United States, 1955-1969. J. Infect. Dis. 122:459-461.

7. Murray, P. R., J. L. Christman, and G. Medofi. 1979. In vitro activity of HR 756, a new cephalosporin, against Neisseria gonorrhoeae. Antimicrob. Agents Chemother. 15:452-454.

8. Philips, C. W., R. D. Allen, and S. N. Cohen. 1976. Penicillinase-producing Neisseria gonorrhoeae. Lancet ii:960.

9. Thornsberry, C., and C. N. Baker. 1979. In vitro antimicrobial activity of piperacillin and seven other $\beta$-lactam antibiotics against Neisseria gonorrhoeae and Haemophilus influenza, including $\beta$-lactamase producing strains. J. Antimicrob. Chemother. 5:137-142.

10. Tjandramaga, T. B., A. Mullie, R. Verbesselt, P. J. Dechepper, and L. Verbist. 1978. Piperacillin: human pharmacokinetics after intravenous and intramuscular administration. Antimicrob. Agents Chemother. 14:829-837. 